\title{
ANÁLISIS DE PROPUESTAS CIUDADANAS EN PRESUPUESTOS PARTICIPATIVOS
}

\section{ANALYSIS OF CITIZEN PROPOSALS IN PARTICIPATORY BUDGETING}

Vicente Barragán Robles Centro Internacional de Estudios Superiores de Comunicación para América

Latina (CIESPAL) vbarragan@ciespal.org

José Manuel Sanz Alcántara Instituto Joaquin Herrera Flores josesanzal@gmail.com

Rafael Romero Instituto Joaquín Herrera Flores

Paelo1@gmail.com

Resumen: Las prácticas de participación ciudadana que pretenden profundizar en la calidad de las democracias están el centro del debate político, fue uno de las reclamaciones principales del movimiento $15 \mathrm{M}$ y de lo que se viene a llamando "nueva política". Una de las prácticas que pueden responder a esta demanda son los presupuestos participativos. El estudio presenta una serie de indicadores que permite analizar de manera comparativa experiencias participativas, desde una visión crítica de la idea de participación, calidad de vida y desarrollo local, con la intención de avanzar más allá de los estudios de caso o de las diferencias en los diseños institucionales. Aporta una metodología de análisis de los procesos, centrándose en las propuestas ciudadanas como punto de partida para conocer los modelos de ciudades que se perfilan tanto desde la ciudadanía como desde las administraciones y ver de este modo las posibilidades de transformación social a través de la elaboración de políticas públicas desde la participación social.

Palabras claves: Democracia, participación, presupuestos participativos, metodología, indicadores, transformación social, calidad de vida, cultura política, ciudad.
Abstract: The Citizen participation practices that pretend to deepen on the quality of democracies are in the center of political debate, it was one of the main claims of the movement $15 \mathrm{M}$ and that is being called "new policy". One of the practices able to respond to this demand are participatory budgets. The study presents a series of indicators that let analyze comparatively participative experiences, from a critical view of the idea of participation, quality of life and local development, with the intention to move beyond case studies or differences in institutional designs. It contributes a methodology of analysis of processes, focusing on citizens' proposals as a starting point to know the cities models that are taking shape as from the citizenship as from the public administrations and see in this way the possibilities of social transformation through the elaboration of public policies from the social participation.

Keywords: Democracy, participation, participatory budgeting methodology, indicators, social transformation, quality of life, political culture, city. 


\section{El análisis de las propuestas ciudadanas como elemento clave de los presupuestos participativos.}

Uno de los puntos de referencia en los que la gran mayoría de los autores están de acuerdo, es en que los Presupuestos Participativos se conciben como una fórmula innovadora en la gestión de los presupuestos locales y, por ende, en la administración de los recursos públicos.

Nos encontramos ante nuevos modelos de gestión que procuran interaccionar de manera más directa con la ciudadanía para la que están destinadas. Las rupturas con los modelos de gestión y los cambios económicos y sociales nos exigen una planificación centrada en otros conceptos. Rosa y Encina (2004) los califican de modelos autónomos o emergentes. Estos modelos proporcionan espacios de articulación y toma de decisiones fuera del Estado y del mercado. El ciudadano toma un papel de participante en la planificación y en la gestión en el ámbito local, trabajando desde un concepto de calidad de vida que une participación y sostenibilidad para un mejor vivir, hacia ciudades no excluyentes, que potencian la variedad y la convivencia desde la complejidad y la diversidad.

Sobre las bases de implementación de un cambio en las relaciones administrativas y proyección de una mejora de la gestión, se impone la idea de la variable participativa. Como comenta Allegretti (2004) la inclusión de la participación es más un medio que un fin para la busca de una administración con intereses más diversos, transparentes y redistributivos. Sin embargo, esta elección ya implica en sí un cambio de perspectiva que impone una creación de espacios paralelos a la propia administración con poder decisorio. Esta condición rompe con la propia rigidez del concepto de representación, ampliando los tiempos y momentos en los que la ciudadanía toma parte del proceso de decisión. Frente a la desafección democrática, se busca una metodología de mayor participación.

Estas dinámicas buscan a la vez reforzar esa actividad autónoma vecinal. Las dinámicas impregnan las estructuras de reforzamiento del tejido asociativo, procurando sacar a estos de su posición clientelar y posicionándolos frente a espacios de decisión colectiva. Se trata de la construcción de unas relaciones que fomenten la interacción de los ciudadanos con la Administración; cuyo fin más ambicioso puede ser el de transformar la estructura social y vital.

Los presupuestos participativos en España asientan ya una práctica. El panorama actual de estos procesos nos muestra experiencias en proceso de inicios a otras ya maduras y asentadas, incluso concluidas. Son diversas las formas en las que en el estado se han ido realizando, y a la vez manteniendo su lazo con la experiencia original de Porto Alegre, cada una ha ido adquiriendo y adaptando sus cualidades propias al contexto local. Sin embargo, al ser herramienta de gestión e implementación participada son necesarios análisis, medición y evaluación continuados como cualquier otra política pública. La posibilidad calidoscópica que ofrece los presupuestos para el análisis es de gran interés. Por este motivo, este equipo investigador desde hace cierto tiempo tenemos puesto nuestro punto de atención en el análisis de las expectativas ciudadanas, y en general la realización de estudios globales de las iniciativas que la ciudadanía solicita a los procesos de gestión participada.

Recordemos a este respecto que las propuestas de la ciudadanía elaboradas y presentadas en los presupuestos participativos nos muestran varios aspectos interesantes en relación a los procesos, a las personas participantes y en sí a los entornos habitacionales. Las propuestas se definen por ser las preferencias de la ciudadanía 
al presupuesto municipal que se pone a disposición de los presupuestos participativos. Por esta razón, se podrían distinguir tres dimensiones básicas: la interna, la subjetiva y la funcional.

Su posición interna viene determinada por ser el canal de comunicación por el cual la población traslada en primera instancia sus preferencias a la administración. Dotan de esta manera a los presupuestos de contenido ya que representan el fin último que es detectar, priorizar, distribuir y ejecutar por medio del gasto público aquellas preferencias que los habitantes de un territorio concluyen como necesarias. En este sentido, como puntualiza Ganuza y Álvarez (2003) los presupuestos participativos se deben entender como un proceso de decisiones compartidas, debiendo permitir reflexionar acerca de los recursos monetarios no como un reparto determinado, ni como una nueva redistribución, sino como un proceso de organización de los recursos existentes con criterios públicos que tiene su canalización por medio de las propuestas.

El momento de presentación y decisión de las propuestas no deja de ser una expresión subjetiva, ya sea de carácter individual o colectivo, debido a que se hace una reflexión y deliberación sobre el lugar que se habita y sus mejoras. Por medio de las propuestas se determina, se hacen saber, o se saca a la luz las necesidades y preferencias de las personas que habitan un lugar en relación a lo que ellos y ellas creen que es mejor para ese sitio. Al ser las propuestas votadas la expresión final del proceso de información y deliberación colectiva, nos acerca a conocer en qué medida la ciudadanía ha podido pasar de ser mera observadora a convertirse en ciudadanos plenos, activos y exigentes con el proceso de presupuestos participativos.

Su dimensión funcional viene acompañada de su capacidad de influencia en la realidad que se habita. Es por ello que se puede afirmar, como lo hace Cabannes (2004), que en muchas de las experiencias de presupuestos participativos -en especial en las latinoamericanas- la ciudadanía ha podido tener mayor acceso a recursos básicos como saneamiento de aguas, pavimentación, transporte público, etc.

En este orden de cosas y desde un punto de vista procedimental las propuestas nos ofrecen información directa de dos elementos claves en el proceso, por medio de las propuestas se constata la voluntad política con el proceso, ya que permiten contrastar la eficacia del proceso por medio de la fiscalización de las inversiones municipales además de dejar constancia de la capacidad que estas tienen para influir en las inversiones municipales (montante monetario, áreas implicadas, propuestas viables, ejecución de las propuestas...).

El análisis de propuesta, viene siendo un trabajo recurrente en nuestro campo de estudio de los presupuestos participativos. Principalmente en la línea de trabajo emprendida con los Presupuestos Participativos de Sevilla y los estudios evaluativos de la calidad democrática de estos (Barragán, Sanz y Romero 2014), nos han llevado a centrarnos en las propuestas como elemento relevante de los procesos de participación ciudadana. Los estudios emprendidos comenzaron en 2004 con la definición de los criterios para la selección de propuestas transformadoras en los presupuestos participativos sevillanos. Estas investigaciones se plasmaron en la evaluación correspondiente del proceso sevillano en 2007. Posteriormente, y como base del trabajo que presentamos a continuación, en el ámbito del estudio comparativo que se realizó en el grupo PARLOCAL (Allegretti et al, 2011) pudimos realizar una comparativa sobre los presupuestos en tres países diferentes: España, República Dominicana y Uruguay. En este contexto, creemos necesario y relevante destacar aquellas conclusiones que pudieron pasar desapercibidas para los casos españoles debido a 
lo amplio del estudio internacional. Realizamos un análisis estatal de los presupuestos, gracias en parte a la información recabada en el estudio mencionado, creímos un deber analizar y presentar como aporte a los procesos emprendidos, además de exponer la metodología que podría ser de utilidad para estudios posteriores sobre procesos de presupuestos participativos.

Para este trabajo, se seleccionaron propuestas presentadas y votadas. Se han codificado y estudiado 1883 propuestas. Son propuestas de al menos dos años de un mismo municipio, sumándose un total de 8 localidades que han implementado presupuestos participativos en España desde el año 2003 al año 2010.

\begin{tabular}{|c|c|c|}
\hline \multicolumn{3}{|c|}{ PRESUPUESTOS PARTICIPATIVOS ANALIZADOS } \\
\hline Propuestas & Procesos & Localidades \\
\hline 1.883 & 16 & 8 \\
\hline
\end{tabular}

Tabla I.- Propuestas, procesos y localidades analizadas

\begin{tabular}{|c|c|c|}
\hline Años & Propuestas & Municipio \\
\hline \multirow{2}{*}{2004} & \multirow{2}{*}{290} & Santa Cristina d’Aro \\
\hline & & Sevilla \\
\hline \multirow{2}{*}{2005} & \multirow{2}{*}{126} & Santa Cristina d’Aro \\
\hline & & Torreperoji \\
\hline 2006 & 100 & St. Cristina d'Aro \\
\hline 2007 & 40 & Torreperojil \\
\hline \multirow{2}{*}{2008} & \multirow{2}{*}{582} & Sevilla \\
\hline & & Getafe \\
\hline \multirow{4}{*}{2009} & \multirow{4}{*}{360} & St. Cristina d'Aro/ / \\
\hline & & Torreperojil/ \\
\hline & & Archidona \\
\hline & & Casabermeja \\
\hline \multirow{4}{*}{2010} & \multirow{4}{*}{385} & Getafe \\
\hline & & Alameda \\
\hline & & Archidona \\
\hline & & Casabermeja \\
\hline
\end{tabular}

Tabla II.- Años de los procesos analizados con propuestas y municipios.

Las conclusiones que presentamos son el resultado de una recogida pormenorizada de propuestas en las localidades participantes. Matriz de datos elaborada a partir de los conocimientos previos del equipo investigador sobre los procesos de presupuestos participativos. En ellas se solicitó descripciones detallada de las propuestas en función de su naturaleza como propuesta ciudadana (nombre, año de votación, ámbito de implementación, proponente/es, descripción...); sus características como elemento clave de los presupuestos participativos (lugar de presentación, año pre- 
supuestario...); así como por su carácter administrativo (inclusión en presupuesto, situación de la ejecución, coste...)

Paralelamente, el estudio ha requerido de una contextualización específica para la codificación y análisis de las propuestas, concluyéndose la necesidad de establecer al menos tres tipos de análisis: uno particular de necesidades ciudadanas básicas, otro de propuestas que generen impacto y uno final que determina la influencia de las propuestas en la cultura política. Estos tres análisis determinan la matriz de codificación al que han sido sometidas las cerca de dos mil propuestas. En los apartados posteriores nos detendremos con mayor concreción.

Finalmente, este análisis se refuerza con un conjunto de entrevistas que se realizaron para el estudio comentado anteriormente de PARLOCAL. Del mismo modo, y como parte de las conclusiones los resultados han sido contrastados con la literatura existente acerca de los presupuestos participativos, tanto desde el punto de vista de su finalidad como desde las evaluaciones existentes.

\section{Marco para definir indicadores para la codificación y aná- lisis de propuestas de presupuestos participativos.}

Un análisis comparativo de las sugerencias presentadas en los procesos de presupuestos participativos requiere un marco amplio que permita la inclusión de todas aquellas iniciativas sugeridas en los procesos. Para ello, se ha estimado conveniente partir al menos de tres ámbitos teóricos: las bases teóricas que sustentan los presupuestos participativos, el concepto de calidad de vida, y la capacidad analítica y evaluativa acerca de las necesidades humanas que ofrece el Enfoque de Desarrollo a Escala Humana (Max-Neef, 1994). Sin embargo, un elemento transversal que recorre todas las experiencias son los entornos metodológicos -particularidades de los presupuestos en cada localidad- y los condicionamientos políticos, sociales y económicos en que se producen.

Los indicadores a la vez tienen para su eficacia que responder a un conjunto bastante extenso de inquietudes, prioridades y necesidades ciudadanas. De este modo, los análisis e indicadores que presentaremos no describen únicamente posibilidades materiales y cuantificables, sino que, en relación con todos los aspectos que convergen en las experiencias de presupuestos participativos, tiene que indagar en las características intangibles que envuelven la propia vida en sociedad. En consecuencia los marcos de reflexión elegidos intercalan elementos de referencia de aceptación mayoritaria, como equipamientos y necesidades básicas; a elementos de la vida en sociedad que requieren de una mayor interpretación, y dependen de mediciones diversas como son las relaciones interpersonales, la convivencia, el ocio, la participación política o la equidad.

Si bien este conjunto diverso nos hace reflexionar sobre estas líneas básicas que engloban la vida en ciudades y pueblos, la principal intención es asentar unas bases que permita ordenar, categorizar y analizar con criterios uniformes el conjunto de propuestas de las diversas experiencias, para, de este modo, permitir un estudio comparativo.

Para comenzar, es imprescindible destacar dos aspectos globales que envuelven estas experiencias participadas, Santos (2003) y Herrera (2005) plantean desde estudios diferentes, una doble distribución característica de los procesos. Una distribución política que debe facilitar el disfrute de los derechos y libertades de todos y 
todas; y, por otro lado; y una distribución a nivel material, o igualdad material, que favorezca el disfrute al conjunto de la ciudadanía de las condiciones económicas, sociales y culturales existentes en la sociedad. De este modo la desigualdad se combate en dos planos el de los derechos políticos y en el de los derechos materiales.

El principio de distribución del poder político parte de la posición ideológica de que la democracia es un proceso de conquistas. Los derechos de participación y los derechos en general, no son derechos otorgados, sino conquistados (Herrera, 2008). Para ello se requiere una cultura y una participación activa de la ciudadanía que llegue a realizar propuestas institucionales y ciudadanas que permitan acceder a los bienes políticos que se propone en el sistema democrático, combinando así las reglas democráticas representativas y participativas. Lo fundamental es la apertura de espacios de decisión, estos son los espacios en los que se tiene que profundizar. De esta forma es necesario un proceso participativo y decisorio que sea ascendente. Por ello, y como consecuencia lógica, los consensos se hacen explícitos. Se desarrolla así un compromiso compartido entre la ciudadanía y la administración (Harrera, op.cit., Santos, op.cit)

Estos enunciados comparten una serie de características básicas que han sido consagradas por las distintas declaraciones internacionales sobre presupuestos participativos (Declaración de Porvenir, Antequera, Málaga, Nairobi, etc.) así como autores muy vinculados a las primeras experiencias latinoamericanas como Souza (2004), Santos (op.cit.) y Cabannes $(2004,2008)$ cuyos principios inspiradores asientan la necesidad de una participación universal, de la apertura de espacios para la generación de ciudadanía, un esquema pedagógico basado en la información y la formación, una voluntad política clara que vincule los presupuestos participativos con un programa de gobierno, y una apuesta por la inclusión basada en una mejora en la estructura distributiva de los recursos públicos.

Las líneas que marcan los presupuestos participativos establecen un campo extenso que destaca desde las necesidades materiales básicas, hasta espacios de construcción de ciudadanía. En este sentido, no sólo se está haciendo alusión a los requerimientos habitacionales básicos de salubridad y medioambientales que representan, en muchos contextos locales, y para colectivos concretos, mejoras en la distribución material de los recursos; o los servicios esenciales para un óptimo bienestar (sanidad, educación, transportes...) sino que también, nos está emplazando a cuestionar la construcción y uso que hacemos de nuestros espacios de socialización (creación de espacios para la socialización y la participación: zonas de recreo, paseos, construcción de centros cívicos y deportivos, ampliación de horarios, mantenimientos de espacios simbólicos, etc.).

Estos aspectos no obstante pueden tener un sustento teórico mayor si partimos de la conceptualización de la calidad de vida. Esta tiene relación directa con la percepción de las vivencias que tiene los sujetos de un entorno. Por ello, este concepto nos remite a un análisis multifuncional en el que se tiene que conocer cómo viven los sujetos, sus condiciones objetivas de existencia y que perspectivas de transformación de estas condiciones desean, así como evaluar el grado de satisfacción que se consigue. De esta forma, la toma en consideración del concepto de calidad de vida es una cuestión que incide directamente en la organización de indicadores que permitan calificar la idea de la vida deseada por una persona. En este ámbito nos encontramos ocho necesidades fundamentales que representan el núcleo de las dimensiones de la vida: bienestar emocional, relaciones interpersonales, bienestar 
material, desarrollo personal, bienestar físico, autodeterminación, inclusión social y derechos (Scharlock y Verdugo, 2003).

Estas premisas nos hacen ver que un alto nivel de vida objetivo depende de distintos factores como recursos económicos, el hábitat, el nivel asistencial o el tiempo libre; y puede ir acompañado de un alto índice de satisfacción individual en bienestar o calidad de vida. Además esta satisfacción viene determinada por el ajuste entre estas características y las expectativas, capacidades y necesidades del individuo, es decir, el sentir de cómo las percibe. Por ello, la segunda premisa definitoria pone el acento en comprender que sería una calidad de vida óptima, y como se estructura sobre la interacción de las personas con su ecosistema que permita un equilibrio tanto en aspectos de sus necesidades como seres vivos como en sus relaciones en lo social, cultural y mitológico (Rueda, S., 1997). Volvemos así a poner el acento en la concepción subjetiva del individuo en relación con sus vivencias de la realidad. Rueda (op. cit) no obstante matiza que estos parámetros al ser subjetivos son también influenciables. El individuo tamiza sus necesidades por medio de expectativas que dependen de las pautas individuales y sociales que a la vez corresponden a una sociedad determinada. Estos componentes subjetivos esenciales del individuo se construyen sobre la base de los matices sociales y colectivos. Por ello la idea de bien común también se ve interceptada por las expectativas personales y colectivas (Castoriadis, 1995). En consecuencia, para la realización de una calidad de vida de una sociedad es imprescindible el establecimiento de un estándar colectivo que sólo es válido para un momento y contexto específico de su establecimiento. Es decir, esta calidad de vida es dinámica, determinada histórica y culturalmente; y que incluye a la vez a ese posible marco ideológico referente o dominante, que complejiza aún más si cabe cualquiera de las soluciones, y dificulta una simple intervención técnica (Rueda, S., op. Cit.).

Los estudios de Desarrollo a Escala Humana (en adelante DEH), abren unos espacios más amplios al contextualizar las necesidades humanas en un marco de transformación que implica a los entornos político, social y económico. Es decir, se favorece ejercitar las estructuras de tránsito desde planos intersubjetivos hacia los planos políticos, en la exigencia de construcción de opciones viables (Encina, J. y Rosa, M., 2004:22). Igualmente estos estudios permiten enmarcar la función de los presupuestos participativos dentro de las prioridades de las personas, así como aporta una clasificación de las necesidades humanas que permite organizar el conjunto de propuestas a analizar. Pero este esquema aporta una perspectiva adicional a tener en cuenta. Nos acerca y define el concepto fundamental de nuestro análisis, que son aquellas propuestas que van más allá de la simple satisfacción de necesidades, para habilitar hacia una transformación mayor, satisfactores sinérgicos en palabras de DEH. No es ya sólo una necesidad de conectar las necesidades humanas con la naturaleza y la tecnología sino que implica también la interacción de los procesos globales y locales, de lo personal y lo social así como la sociedad civil y el Estado (DEH, Op. cit.).

De esta forma el enfoque se cuestiona acerca de los niveles de vida que deben garantizarse universalmente para permitir a todos los ciudadanos y ciudadanas participar en la vida social. Proponiendo asimismo un debate social acerca de lo que es básico, secundario o de lujo en las necesidades humanos, e interpela sobre las 
actuaciones inaceptables por los efectos sociales y ambientales que suponen (Riechmann, J, 2006: 65-70) ${ }^{1}$.

En este marco, los EDH, como también expresaban los presupuestos participativos, ponen su acento en transformar a la persona objeto en persona sujeto de su propio desarrollo. El protagonismo se traslada a las personas y no a los actores económicos únicamente. Privilegia de esta forma tanto la diversidad como la autonomía, en el que el protagonismo del sujeto sobre el espacio que habita sea realmente posible. En consecuencia, es prioritario trabajar a la vez en una profundización democrática que destruya dinámicas paternalistas, y active y estimule esquemas de soluciones creativas que vayan de abajo hacia arriba. Por esta razón las necesidades no se entienden exclusivamente en un esquema de carencia, sino también sobre una idea de potencia, de activadores y movilizadores de la ciudadanía a favor de la resolución de sus problemas. Resaltando de este modo su aspecto personal y a la vez colectivo.

Uno de los elementos esenciales de este esquema analítico es el de no examinar las necesidades, y en consecuencia la satisfacción de éstas, de forma estática y unívoca. Sino que introduce una serie de variables que permiten comprender el conjunto de categorías que mueven al ser humano a favor de su bienestar por una serie de estrategias que pueden mejorar o empeorar su situación. Y estas estrategias a la vez, no dependen exclusivamente de un crecimiento económico y material sino que avanza y las necesidades terminan ampliando la perspectiva y trasvasa la idea de satisfacción por medio de bienes y servicios para ampliar la perspectiva y relacionarla con prácticas sociales, formas de organización, modelos políticos y valores que repercuten sobre las formas en que se expresan las necesidades (Max-Neef, 1994).

Esta referencia nos abre un espacio importante en nuestro trabajo ya que en el propio proceso de desarrollo es el elemento de arranque. De esta forma, lo importante no es ya sólo el conseguir las metas de satisfacción de esas necesidades fundamentales, sino que el propio proceso impulse desde su comienzo hasta el fin todo un conjunto de mejoras necesarias. "O sea, que la realización de las necesidades no sea la meta, sino el motor del desarrollo, sea capaz de estimular permanentemente la generación de satisfactores sinérgicos" (Max-Neef, M.A., 1994:136).

Llegado a este punto, podemos construir una aproximación objetiva a la calidad de vida que depende de los siguientes variables de interés o preocupación:

- Aspectos decisivos para el bienestar general de las personas: trabajo, educación, sanidad, vivienda y equipamientos.

- Aspectos relacionados con el medio, con la calidad ambiental: calidad ambiente atmosférica, el ruido, la calidad del agua, etc.

- Aspectos relacionados con los aspectos psicosociales fundamentalmente del ámbito interactivo de los sujetos: relaciones familiares, relaciones interpersonales, ocio, tiempo libre, etc.

- Aspectos referentes al orden socio-político: participación social, la seguridad personal y jurídica, etc.

1 Riechmann hace referencia a la conferencia de Albert Recio titulada "Empleo y medio ambiente: necesidades y dificultades de un proyecto alternativo" ponencia en el curso de verano de la UCM "Nuevas economías: una alternativa ecológica". San Lorenzo del escorial, 19 a 23 de julio de 2004. 
Las dinámicas, objetivos y fines de los presupuestos participativos son, como plantea el DEH, un satisfactor sinérgico en sí. O por lo menos, planta las bases para colmar las necesidades de forma sinérgica. Sin embargo, y es lo que intentamos analizar en este trabajo, es imprescindible conocer si por medio las metodologías de presupuestos participativos permiten transformaciones más allá de la satisfacción de necesidades, si se produce, como planteaba Castoriadis (2006) cambios en los imaginarios colectivos, en las prácticas y valores que cuestionen los referentes dominantes.

\begin{tabular}{|c|c|c|c|}
\hline \multicolumn{4}{|c|}{ Resumen general del marco teórico y su relación con las dimensiones y variables seleccionadas. } \\
\hline Indicadores & Pre. Participativos & Calidad de vida & DEH \\
\hline Habitabilidad & $\begin{array}{l}\text { Favorecen una trasfor- } \\
\text { mación compartida del } \\
\text { entorno. }\end{array}$ & $\begin{array}{l}\text { Su mejora tiene relación } \\
\text { directa con necesidades } \\
\text { básicas y la accesibilidad } \\
\text { a equipamientos y mejoras } \\
\text { del entorno. }\end{array}$ & $\begin{array}{l}\text { Las necesidades son } \\
\text { cubiertas por medio de } \\
\text { decisiones referentes al } \\
\text { entorno y las situaciones } \\
\text { vitales. }\end{array}$ \\
\hline Movilidad & $\begin{array}{l}\text { El acceso universal a los } \\
\text { recursos y equipamientos } \\
\text { requieren de las posibilida- } \\
\text { des de desplazamiento. }\end{array}$ & $\begin{array}{l}\text { Los desplazamien- } \\
\text { tos son necesidades } \\
\text { fundamentales. } \\
\text { El debate actual relaciona } \\
\text { este indicador con el desa- } \\
\text { rrollo sustentable. }\end{array}$ & $\begin{array}{l}\text { Favorece los aspectos } \\
\text { relacionados con la libertad } \\
\text { y el acceso a recursos. }\end{array}$ \\
\hline $\begin{array}{l}\text { Espacios } \\
\text { verdes }\end{array}$ & & $\begin{array}{c}\text { Calidad ambiental como } \\
\text { parte de las necesidades } \\
\text { humanas. }\end{array}$ & $\begin{array}{l}\text { Medio ambiente saludable } \\
\text { como satisfactor sinérgico. }\end{array}$ \\
\hline $\begin{array}{l}\text { Limpieza y } \\
\text { salubridad }\end{array}$ & $\begin{array}{l}\text { Las mejoras en salubri- } \\
\text { dad miden distribución de } \\
\text { recursos. }\end{array}$ & $\begin{array}{l}\text { Se miden umbrales de me- } \\
\text { jora en la calidad de vida }\end{array}$ & $\begin{array}{l}\text { Son recursos básicos que } \\
\text { impulsan otras mejoras. }\end{array}$ \\
\hline $\begin{array}{l}\text { Equipamien- } \\
\text { tos }\end{array}$ & $\begin{array}{l}\text { La inclusión, la distribución } \\
\text { y la participación dependen } \\
\text { de este indicador. }\end{array}$ & $\begin{array}{l}\text { Miden calidades de vida y } \\
\text { recursos distribuidos. }\end{array}$ & $\begin{array}{l}\text { Recursos y mejoras que } \\
\text { pueden fundamentar avan- } \\
\text { ces. No obstante requieren } \\
\text { de análisis exhaustivos. }\end{array}$ \\
\hline Cultura & $\begin{array}{l}\text { Forma parte de los } \\
\text { recursos materiales a ser } \\
\text { distribuidos. }\end{array}$ & $\begin{array}{c}\text { Parte básica de las necesi- } \\
\text { dades fundamentales }\end{array}$ & $\begin{array}{l}\text { Necesidad básica que } \\
\text { requiere del estudio de los } \\
\text { satisfactores activados. }\end{array}$ \\
\hline Salud & $\begin{array}{l}\text { Es también un indicador de } \\
\text { redistribución e inclusión. }\end{array}$ & $\begin{array}{l}\text { Bienestar como necesidad } \\
\text { fundamental. }\end{array}$ & $\begin{array}{c}\text { Una salud óptima posibilita } \\
\text { otras metas. }\end{array}$ \\
\hline Cogestión & $\begin{array}{l}\text { La corresponsabilidad es } \\
\text { una finalidad. }\end{array}$ & $\begin{array}{l}\text { La participación es una } \\
\text { necesidad personal y } \\
\text { colectiva. }\end{array}$ & $\begin{array}{l}\text { Una participación más } \\
\text { activa de la ciudadanía } \\
\text { promueve otros valores y } \\
\text { potencia conocimientos } \\
\text { colectivos. }\end{array}$ \\
\hline $\begin{array}{l}\text { Integración } \\
\text { social }\end{array}$ & $\begin{array}{l}\text { Es parte esencial para } \\
\text { cumplir la participación } \\
\text { universal. }\end{array}$ & $\begin{array}{c}\text { Necesidad básica personal: } \\
\text { libertad y autoestima. }\end{array}$ & $\begin{array}{l}\text { Si en el centro del desa- } \\
\text { rrollo está la persona, este } \\
\text { indicador es un fin. }\end{array}$ \\
\hline
\end{tabular}




\begin{tabular}{|c|c|c|c|}
\hline $\begin{array}{c}\text { Espacios } \\
\text { socialización }\end{array}$ & $\begin{array}{l}\text { Se debe generar espacios } \\
\text { ciudadano. }\end{array}$ & $\begin{array}{l}\text { Interacción social es otra de } \\
\text { las necesidades básicas. }\end{array}$ & \\
\hline $\begin{array}{l}\text { Comuni- } \\
\text { cación y } \\
\text { Ciudadanía }\end{array}$ & $\begin{array}{l}\text { La información, la pedago- } \\
\text { gía y la reflexión requieren } \\
\text { de estas herramientas. }\end{array}$ & $\begin{array}{c}\text { Es necesario desarrollar } \\
\text { los aspectos de identidad y } \\
\text { participación }\end{array}$ & $\begin{array}{l}\text { Son satisfactores de ser, de } \\
\text { identidad y participación }\end{array}$ \\
\hline $\begin{array}{l}\text { Más allá } \\
\text { localidad }\end{array}$ & $\begin{array}{l}\text { No sólo se crea conciencia } \\
\text { sobre el entorno sino sobre } \\
\text { el exterior }\end{array}$ & & $\begin{array}{l}\text { La empatía e identificación } \\
\text { con los otros/as es un } \\
\text { satisfactor sinérgico }\end{array}$ \\
\hline Empleo & $\begin{array}{l}\text { Marcan posibilidades de } \\
\text { desarrollo personal }\end{array}$ & Necesidad fundamental & $\begin{array}{l}\text { Con matices, satisface } \\
\text { hacer y creación }\end{array}$ \\
\hline Educación & $\begin{array}{l}\text { Marcan posibilidades de de- } \\
\text { sarrollo personal, colectivo } \\
\text { e inclusión }\end{array}$ & $\begin{array}{c}\text { Necesidad básica colectiva } \\
\text { y personal }\end{array}$ & $\begin{array}{c}\text { Su desarrollo forma } \\
\text { parte de la necesidad de } \\
\text { entendimiento }\end{array}$ \\
\hline Género & $\begin{array}{l}\text { Los grupos excluidos o } \\
\text { apartados son población de } \\
\text { referencia. }\end{array}$ & $\begin{array}{l}\text { Necesidad básica personal: } \\
\text { libertad y autoestima. }\end{array}$ & $\begin{array}{l}\text { La igualdad de derechos es } \\
\text { un satisfactor de la libertad } \\
\text { y el tener. }\end{array}$ \\
\hline
\end{tabular}

Tabla III.- Dimensiones y variables de análisis en relación al marco teórico. Elaboración propia.

\section{Los tres análisis}

como planteábamos en puntos anteriores, proponemos un estudio de propuestas atendiendo a tres análisis específicos que permiten relacionar las sugerencias ciudadanas con los fundamentos teóricos apuntados anteriormente:

\subsection{Análisis de la calidad de vida: necesidades y preferencias de la población en los aspectos habitacionales, medioambientales y necesidades sociales}

Las propuestas concretan las demandas ciudadanas definiendo las prioridades de gastos; a partir de su estudio obtenemos información acerca de las necesidades sentidas por una determinada población, pero también sobre las preferencias de esta población respecto a las características de su entorno, lo que perfila un modelo de desarrollo urbano y convivencial ${ }^{2}$, es decir, un modelo de ciudad determinado.

\subsection{Análisis del impacto en la política municipal.}

Atendiendo al volumen de su dotación económica, a su relación con respecto al total del presupuesto municipal, y al nivel de ejecución de las propuestas podemos dar cuenta de los puntos clave que determinan la posibilidad y el sentido de los presupuestos participativos: compromiso político de las administraciones; empoderamiento de la ciudadanía; aumento de la justicia social ${ }^{3}$.

2 Se utiliza aquí el adjetivo planteado por Ivan Illich cuando expresa que la sociedad covivencial será aquella que promueva estructuras que permitan una mayor encuentro con lo común y con el medio que nos rodea (Illich, I., 1973)

3 Nos referimos en este ámbito a los criterios establecidos en gran parte de los procesos de introducir criterios concretos para alteración del orden de priorización de las propuestas tras la votación de estas en función de indicadores socioeconómicos. 


\subsection{Análisis de la cultura política, cambios y evolución de los presupuestos participativos y de la participación ciudadana}

Los presupuestos participativos suponen una apuesta por la mejora de la administración, la inclusión de la ciudadanía en la reflexión acerca del destino del presupuesto municipal y la promoción una gestión más eficaz de los recursos, con el fin de mejorar la relación entre gobernantes y gobernados al aumentar la transparencia y eliminar las relaciones clientelares. Paralelamente entre sus objetivos encontramos el de profundizar en la democracia a través del fomento de una ciudadanía activa, que sea capaz de participar en la dirección de los asuntos públicos y asuma paulatinamente mayores cuotas de poder político.

A este respecto es importante señalar que en la mayoría de las experiencias de presupuestos participativos, al contrario de lo sucedido en el caso original de Porto Alegre, su puesta en marcha no parten de una exigencia de la ciudadanía, sino que depende de la iniciativa de un gobierno local, o incluso al impulso personal de alguno de sus miembros (Fedozzi, L., 1998:236-244). Con esto queremos señalar que en la mayoría de las ocasiones nos encontramos ante una situación de partida en las que el tejido asociativo es muy débil, y su desarrollo va a depender en buena medida del compromiso real y el buen hacer del gobierno municipal (Sintomer, Y., 2001; Ganuza, E.,2003).

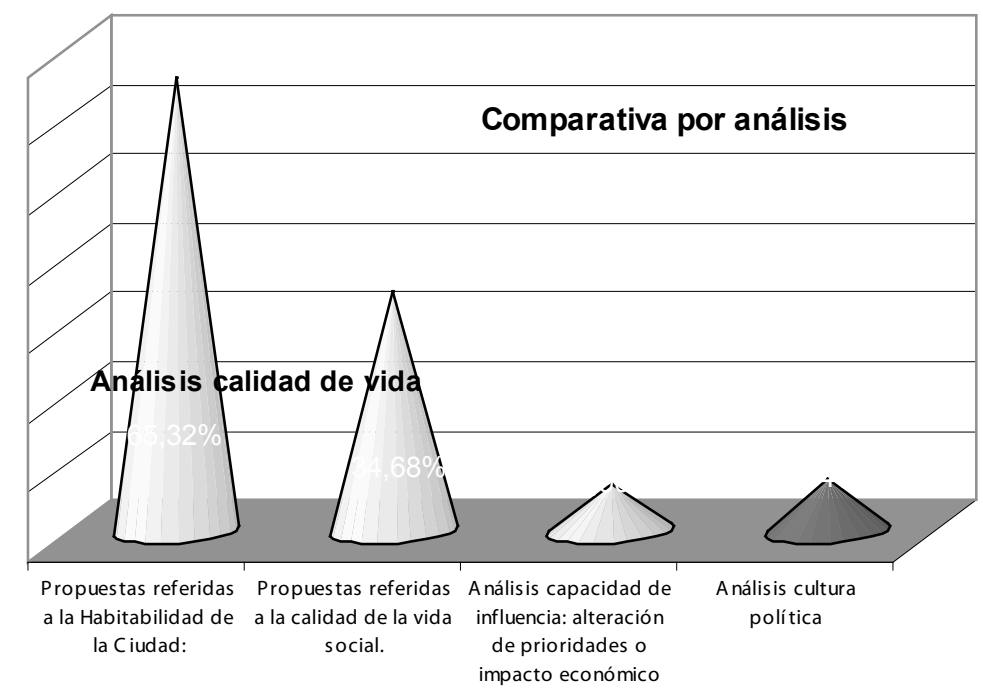

Gráficos 1.- Análisis comparado de las propuestas en presupuestos participativos de municipios analizados. Elaboración propia.

Como señaláramos en párrafos anteriores, los presupuestos participativos no son una metodología cerrada, en su formulación original se incluye la pretensión de su crecimiento y ampliación, pero este desarrollo no se da necesariamente. Es decir, a partir de su puesta en funcionamiento los presupuestos participativos pueden evolucionar hacia el objetivo de crear una ciudadanía organizada, activa y capaz, o por el contrario pueden quedar restringidos a la mera gestión de lo micro (a nivel de los problemas de calle o de barrio) sin mayor repercusión en la política municipal. 
Una experiencia de presupuesto participativo que evolucionara hacia una mayor importancia de la participación en las decisiones de la política municipal se correspondería con las siguientes tendencias observable a partir del análisis de las propuestas:

- Tendencia al aumento de su importancia presupuestaria.

- Nivel de ejecución de propuestas cercano al total.

- Tendencia a la desaparición de propuestas referidas a necesidades básicas.

- Tendencia al aumento del número o importancia económica del tipo de propuestas que se refieran a toda la ciudad en contraposición con aquellas que se centran en ámbitos de interés más reducidos como la calle o el barrio.

- Tendencia al aumento del número o importancia económica del tipo de propuestas que se refieran a obras nuevas en contraposición con aquellas referidas a obras de reparación y mantenimiento.

- Tendencia al aumento de las propuestas que posean un contenido político que no se restrinja a la distribución de los recursos económicos. O propuestas referidas al modelo de desarrollo urbano, o a cambios en las relaciones sociales, en la moral o en las normas.

Así pues, el análisis de las propuestas puede darnos claves para interpretar el nivel de evolución de una práctica concreta de presupuestos participativos así como análisis comparativo con otras experiencias, como es el caso que nos ocupa, como mediante un análisis diacrónico tomando como referencia la misma experiencia en su inicio ${ }^{4}$ :

\begin{tabular}{|c|c|c|}
\hline Análisis y dimensiones del e & de las propuestas ciudad & n presupuestos participativos \\
\hline \multirow{6}{*}{ Análisis de la calidad de vida. } & Habitabilidad de la ciudad & $\begin{array}{l}\text { Estructura espacial (movilidad y } \\
\text { accesibilidad) }\end{array}$ \\
\hline & Calidad de la vida social & $\begin{array}{l}\text { Espacios públicos y espacios de } \\
\text { socialización }\end{array}$ \\
\hline & \multirow{4}{*}{ Calidad medioambiental } & Calidad Medio ambiental/ \\
\hline & & Salubridad entorno urbano/ \\
\hline & & $\begin{array}{l}\text { Iniciativas medioambientales y de } \\
\text { protección de espacios. }\end{array}$ \\
\hline & & $\begin{array}{c}\text { Prevención de catástrofes } \\
\text { naturales. }\end{array}$ \\
\hline $\begin{array}{l}\text { Análisis sobre capacidad de } \\
\text { influencia y la alteración de } \\
\text { prioridades o impacto social } \\
\text { y/o económico }\end{array}$ & Equipamientos & $\begin{array}{l}\text { Equipamientos básicos y equipa- } \\
\text { mientos de relevancia socioeco- } \\
\text { nómica para la comunidad }\end{array}$ \\
\hline
\end{tabular}

4 En el estudio original "Análisis de las expectativas ciudadanas expresadas en las propuestas de presupuestos participativos" (Barragán, Romero y Sanz, 2012) la persona que lo desee puede encontrar un análisis más exhaustivo de las variables e indicadores que hemos apuntado y que se detallan en el cuadro resumen. 
Análisis sobre los cambios y avances en la cultura política
Ampliación y profundización en democracia y en la vida política y social en distintos niveles
Propuestas de contenido político local y más allá de la localidad.

Cuestiones de género.

Otras cuestiones relacionadas con la transparencia institucional, la participación y la relaciones ciudadanía e instituciones de gobierno.

Tabla IV.- Análisis, dimensiones e indicadores utilizados. Elaboración propia.

\section{Estudio comparado de las propuestas en las localidades españolas seleccionadas.}

En el caso del estudio que presentamos, se analizaron ocho experiencias locales, cinco son en municipios rurales, todos ellos con menos de 20.000 habitantes, dos son ciudades medias que superan los 100 mil habitantes, y una gran ciudad con más de 700 mil. Solamente dos localidades no pertenecen a la región andaluza (Getafe y Santa Cristina d’Aro). Entre todas ellas se dan grandes diferencias tanto presupuestarias como de población.

Los procesos analizados muestran como las propuestas hacen referencia mayoritariamente a aspectos relacionados con la calidad de vida. Generalmente las propuestas hacen referencia a la organización del espacio urbano y la calidad medioambiental. El indicador más puntuado es el relacionado con la movilidad. Del mismo modo, encontramos propuestas que inciden en la mejora de los espacios públicos y comunes. En lo que respecta a medio ambiente las propuestas se dividen entre aquellas que tratan temas de salubridad ambiental, y aquellas que pretenden la provisión de espacios verdes y mejoras ambientales.

\begin{tabular}{|c|c|c|c|}
\hline Municipios & $\begin{array}{c}\text { P. municipal } \\
\text { per cápita }\end{array}$ & $\begin{array}{c}\text { Presupuesto municipal } \\
\mathbf{( 2 0 0 9 )}\end{array}$ & $\begin{array}{c}\text { Población } \\
\text { (2009) }\end{array}$ \\
\hline Santa Cristina & $2.203,74 €$ & $10.203 .304 €$ & 4.630 \\
\hline Sevilla & $1.466,25 €$ & $1.032 .528 .058 €$ & 704.198 \\
\hline Archidona & $1.045,68 €$ & $9.240 .703 €$ & 8.837 \\
\hline Casa Bermeja & $960,49 €$ & $3.413 .580 €$ & 3.554 \\
\hline Alameda & $941,91 €$ & $5.110 .786 €$ & 5.426 \\
\hline Torreperogil & $736,64 €$ & $10.395 .429 €$ & 14.112 \\
\hline
\end{tabular}

Tabla V.-Renta per cápita, presupuesto y población de los municipios analizados a 2009.

Elaboración propia.

Las conclusiones del estudio muestran que los presupuestos participativos analizados tienen una marcada tendencia hacia la subsanación de requerimientos básicos del entorno urbano. Los indicadores que predominan son los relacionados con la habitabilidad de la ciudad y, en concreto, aquellos con incidencia directa en la me- 
jora del entorno más cercano (calles, plazas o barrios). En cambio, las propuestas cuya finalidad son necesidades básicas de la población no tienen una presencia significativa.

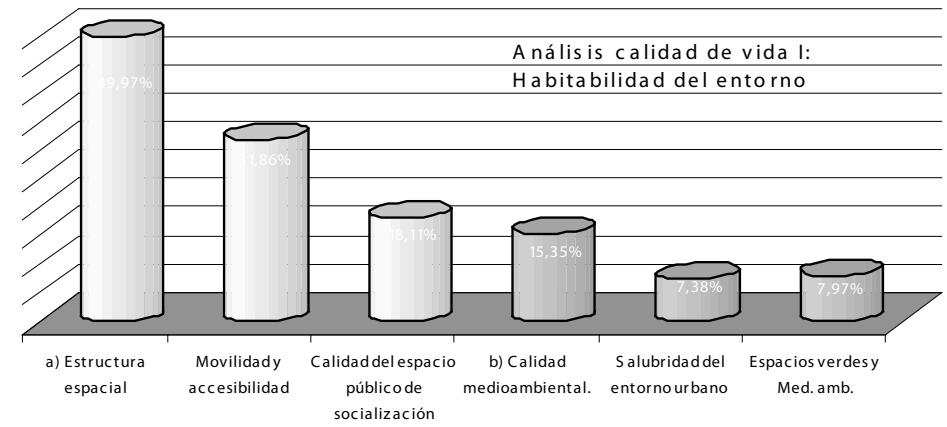

Total propuestas de calidad de vida. - El $100 \%$ de las propuestas puntúa alguno de estos indicadores.

Total de propuestas Habitabilidad.- 1.230 propuestas, el $65,2 \%$ del total de propuestas.

Total de propuestas Calidad de vida social. - 653 propuestas, el $34,68 \%$ del total de propuestas.

Gráficos 2.- Análisis del Grupo primero dedicado a la calidad de vida: habitabilidad y medio ambiente. Elaboración propia.

En este orden de cosas, el segundo grupo que más propuestas recoge es aquel que mide la calidad de vida desde el punto de vista colectivo (educación, sanidad, empleo, etc.). Destacando las propuestas que fomentan un entorno sociocultural diversos. Estas últimas propuestas tienen una relación directa con una característica propia de los presupuestos participativos españoles que es la de abrir un espacio en estos para la petición de actividades socioculturales diversas que normalmente están relacionadas con el área de participación ciudadana y cultura. Otras propuestas aunque con menor representación se sitúan en el ámbito de la educación y de la participación.

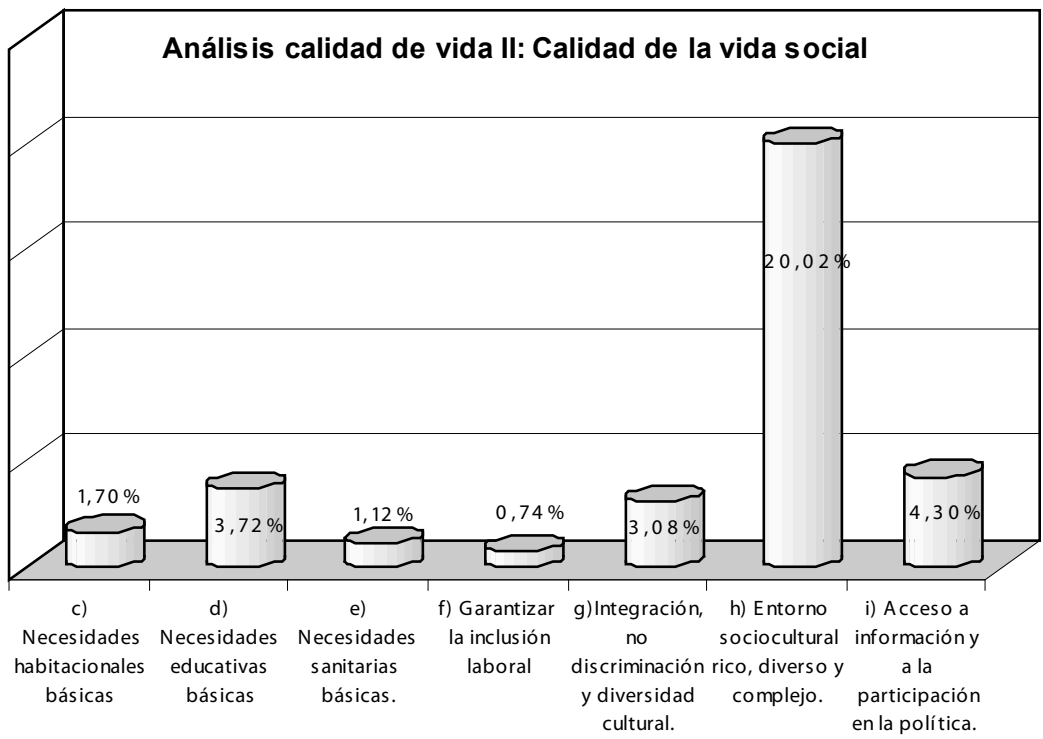

Gráficos 3.- Análisis del Grupo primero dedicado a la calidad de vida: necesidades colectivas. Elaboración propia. 
Las características concretas de los presupuestos participativos, ya sea por la baja importancia de estos en los programas políticos o ya sea por la reducida capacidad decisoria en recursos que estos procesos tienen, observan como la gran mayoría de propuestas se refieren a inversiones de escasa envergadura. Es por ello que, aun a falta de un análisis más exhaustivo en lo que a ejecución se refiere, los presupuestos muestran una clara línea orientada a inversiones de reparación y mantenimiento de la estructura espacial de la ciudad.

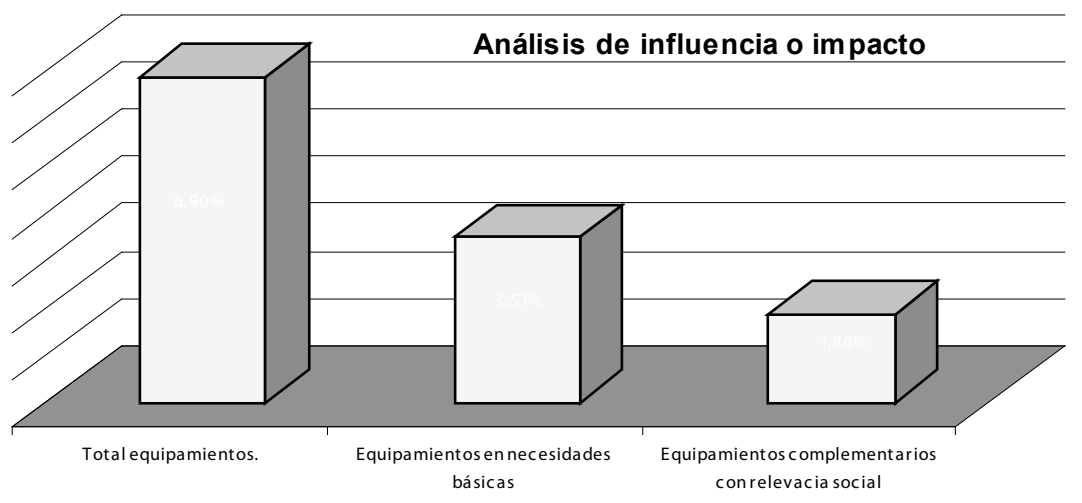

Gráficos 4.- Análisis grupo segundo: equipamientos de importancia económica y/o impacto social. Elaboración propia.

Los resultados de estos dos análisis muestran una tendencia generalizada en los presupuestos participativos que viene referida por las limitaciones que estos procesos encuentran en la propia estructura administrativa. Las experiencias, ya estén en estados iniciales o sean procesos maduros incluso finalizados suelen estar ubicados en las áreas de participación ciudadana de las corporaciones locales. Áreas que por lo general tienen una importancia limitada en las estructuras de los gobiernos locales. Además, las experiencias habitualmente se conforman entorno a disponibilidades presupuestarias que las diferentes delegaciones de gobierno ponen a disposición de los procesos. Observamos en estas experiencias ciertos recelos ante posibles resultados no deseados. De igual modo, bien es cierto que en los procesos más asentados estos recelos van limándose. No obstante, el resultado por lo general es que los presupuestos participativos suelen tener una capacidad limitada de decisión. Esto muestra no obstante que este tipo de experiencias cuenta con una voluntad política restringida, en la que las decisiones de carácter transversal, las aportaciones de mayor importancia no están incluidas.

En este sentido, las experiencias se ven bastante influenciadas por las propias estructuras locales y lo que piensan sobre los presupuestos. Y del mismo modo, y en descarga de los gobiernos locales, también existen unas dificultades intrínsecas relacionadas con las competencias locales y todo el esquema administrativo que influyen en estas posibilidades decisorias. Sin embargo, en la actualidad y salvo escasas excepciones, las posibilidades de una inmersión mayor en asuntos más complejos, con carácter transversal, horizontal y mayor capacidad de influencia tanto económica como planificadora de los gobiernos locales no están siendo tomadas en cuenta. La madurez de los presupuestos como hemos comentado, y como atestiguan Allegretti (2011) y Cabannes (2008) pasa por abrir estos espacios de mayor influencia, al igual 
que se ha realizado con otros procesos participativos, sin ser vinculantes, como se ha realizado con los planes urbanísticos y los planes estratégicos en distintas ciudades.

Dicho de otro modo, los resultados atestiguan lo ya expresado de manera teórica por la literatura en lo referente a las experiencias españolas y es que estos tienen como objetivo general incidir en la transparencia de la gestión, en un ensayo de fortalecimiento de las reglas democráticas por medio de la apertura de la participación a los mecanismos de democracia representativa. Aportando en algunos casos intentos de mejora de la participación ciudadana fortaleciendo el tejido social y asociativo, procurando la inclusión e incidir la redistribución de recursos.

Estas ideas quedan reforzadas con el tercer análisis que introducimos. El análisis de cultura política nos presenta una escasa incidencia de propuestas que tengan esta finalidad. Aunque esto no es óbice para que todas los indicadores estén representados (cogestión ciudadana, propuestas que van más allá de lo política local o las relacionadas con género). No obstante estas no se distribuyen de igual forma. Los municipios más pequeños concentran sus peticiones en el ámbito de la propuestas de espacio ciudadanos y de participación. Los más poblados tienen cabida propuestas relacionadas con la solidaridad internacional y la cooperación.

En lo que respecta a género, siendo los presupuestos participativos procesos marcadamente feminizados en su participación (como atestigua toda la literatura relacionada), son los municipios con mayor densidad poblacional los que recogen propuestas más concretas relacionadas con cambios culturales y de reivindicación identitarias. Destaca en este indicador el municipio de Sevilla que abrió espacio en este ámbito para cuestiones relacionadas con los movimientos LGTB $^{5}$. El caso de Sevilla, sin embargo, es representativo en este aspecto pues recoge el doble de propuestas en este indicador que el número total de los otros municipios todos sumados.

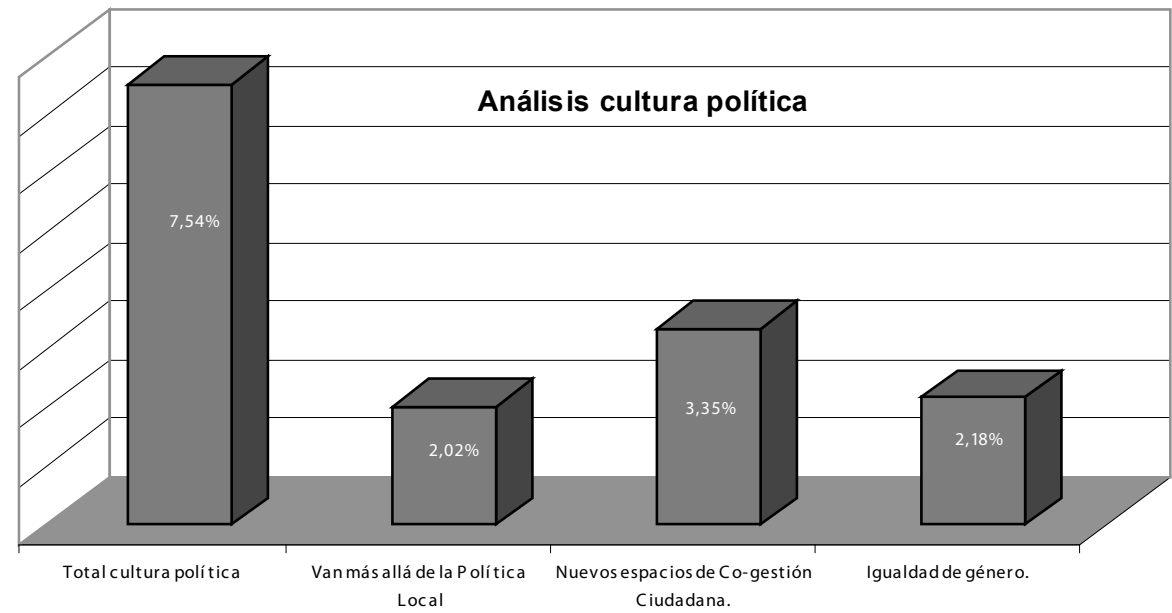

Gráficos 5.- Análisis grupo tercero: Cambios en la cultura política. Elaboración propia.

Un elemento a destacar y concretar en este tipo de análisis y el que hemos observado tienen cierta relevancia para el análisis de propuestas en los presupuestos participativos es el de realizar los análisis introduciendo el eje rural/urbano, ya que,

5 Acrónimo para representar al colectivo formado por Lesbianas, Gays, Transexuales y Bisexuales. 
aunque los presupuestos tienen tendencias parecidas, este eje permite establecer diferencias relevantes.

\begin{tabular}{|l|c|c|c|c|c|}
\hline \multicolumn{2}{|c|}{ Total de propuestas } & Rural & $\%$ & Urbano & $\%$ \\
\hline \multicolumn{2}{|c|}{1.842} & 994 & & 848 & \\
\hline Actividades & 635 & 240 & $24,14 \%$ & 395 & $46,58 \%$ \\
\hline Inversiones & 1.207 & 754 & $75,86 \%$ & 453 & $53,42 \%$ \\
\hline
\end{tabular}

Tabla VI.- Resultados en función eje rural/urbano. Elaboración propia.

Los presupuestos participativos de los municipios rurales constatan una tendencia hacia las propuestas referidas a inversiones en infraestructuras, concentran dos tercios del total. Frente a las ciudades que equilibran sus inversiones en infraestructuras y actividades.

Respecto a estas inversiones en infraestructuras, las reparaciones y mejora de equipamientos son el $80 \%$ de las inversiones en los municipios de ámbito rural. Frente a un $97 \%$ en el caso urbano. Con respecto a las propuestas referidas a grande obras de equipamiento del tipo de equipamientos deportivos, parques o centros cívicos, en ambos tipos de municipios el porcentaje es similar e inferior al 4\%. Donde sí se observa diferencias es en el número de propuestas referidas a subsanar las carencias de equipamientos que atiendan a cubrir necesidades básicas; propuestas que se concentran en las categorías de movilidad y salubridad. En los municipios de ámbito rural este tipo de propuestas concentran casi el $9 \%$; reduciéndose a menos del $0,5 \%$ en el ámbito urbano. En concreto, la mayor diferencia se concentra en equipamientos básicos de salubridad donde el número de propuestas en los municipios rurales llega a ser trece veces superior a las registradas en las ciudades.

\begin{tabular}{|c|c|c|}
\hline Inversiones & Rural & Urbano \\
\hline Necesidades básicas & $8,49 \%$ & $0,44 \%$ \\
\hline Grandes obras & $3,45 \%$ & $2,65 \%$ \\
\hline Arreglos y mejoras & $88,06 \%$ & $96,91 \%$ \\
\hline
\end{tabular}

Tabla VII.- Tipo de inversiones en el eje rural/urbano. Elaboración propia.

En el mismo orden de cosas, las propuestas rurales de calidad de vida social tienen una puntuación diferenciadamente más baja que las de los espacios urbanos. Estos datos dan cuenta de la mayor necesidad un espacio público habitable que permitan las relaciones sociales en unos entornos, los urbanos, de alta densidad de población y producto de un rápido crecimiento en los que a la hora de ordenar su urbanización.

Por otro lado, las propuestas de actividades se centran, en propuestas referidas a espacios de socialización y a cultura, aun así se observan diferencias entre ambos tipos de poblaciones dado que mientras en las ciudades la mayoría de propuestas puntúan en estas dos categorías siendo muy inferior el número de las que puntúan en el resto, en cambio, en las poblaciones rurales las puntuaciones están mucho más distribuidas teniendo también relevancia el número de actividades que puntúan en las categorías de movilidad, ciudadanía y comunicación, y medio ambiente.

Las diferencias respecto a los intereses y necesidades sentidas por los ciudadanos en ambos ámbitos quedan también patentes en el momento de establecer la tercera categoría en importancia según el número de propuestas que en ella puntúan. 
Mientras en el ámbito urbano esta categoría es la de propuestas referidas a cultura, con una diferencia de 20 puntos porcentuales frente a los pequeños municipios, cuyas preferencias estriban en limpieza y salubridad, siendo estas menores en las ciudades.

Por último, en las zonas urbanas las propuestas de integración social son más del doble que en las zonas rurales.

\section{Conclusiones globales entorno a capacidad de transfor- mación y limitaciones de los presupuestos españoles.}

Los análisis expuestos confirman lo adelantado por ahora para los procesos españoles analizados en este estudio. Son procesos en los que la voluntad política ha estado focalizada en la apertura de espacios para la decisión compartida de recursos cotidianos de las competencias locales.

El marco de los presupuestos participativos analizados centra sus llamamientos a una extensión de la democracia representativa con una ampliación de la participación, con la clara intención de acercar las decisiones del poder institucional a la ciudadanía, en favor de una mayor transparencia. El paradigma base de las experiencias es, de este modo, la de hacer una gestión pública, una distribución de los (algunos) recursos públicos de forma participada implicando esta un mayor control y fiscalización. El esquema democrático hace patente así que necesita de una ampliación de espacios decisorios que a la vez reconozca a la ciudadanía como interlocutora y finalista de las decisiones políticas de gestión. Por ello, al ser los afectados, su palabra se hace ley.

En general se puede afirmar que se ha puesto sobre la arena política y ciudadana la doble distribución (política y material) que nos comentaba el profesor Herrera. Efectivamente se han abierto espacios de decisión y toma compartida de pareceres.

En general, más por el proceso que por las propias propuestas, se entiende que los presupuestos han influido en la efectividad de las políticas municipales acercando a la comunidad la posibilidad de acordar de forma conjunta las prioridades de la comunidad. Igualmente, se observa cierta mejora en la capacidad propositiva de la población, entendiendo que una vez introducidos los presupuestos participativos la ciudadanía ha tenido un espacio abierto para demandas veladas por las prácticas representativas. En cierto modo, la población experimenta autonomía propositiva y abre espacios para solventar sus necesidades, en especial aquellas que tienen que ver con aspectos de recursos y equipamientos primarios.

Sin embargo, realizado nuestro estudio de las propuestas y las entrevistas, nos parece ineludible, con el fin de completar nuestro trabajo, hacer una serie de reflexiones que parte de la siguiente observación general.

Apreciamos que las peticiones realizadas por la ciudadanía, las propuestas presentadas y votadas, son consecuencias mayormente de un intento de mejorar el reparto real de los recursos (voluntad política). Pero que, aunque introduce aspectos participativos, no son consecuencias directas y exclusivas de un proceso de cambio participativo más profundo. En otras palabras, lo que nos ha extrañado es que aun analizándose un número significativo de propuestas, dentro de experiencias claramente diferenciadas, y con una cantidad también importante de localidades implicadas, el análisis de las expectativas ciudadanas -las propuestas presentadas 
y votadas- presenta muy pocas propuestas que se salgan del guion marcado. El conjunto de los procesos -aunque con las consecuentes diferencias y desviaciones según las localidades están fundamentalmente centrados en establecer dónde y a quiénes se deben dirigir los recursos cotidianos, más que en cualquier otra decisión de calado político-. En otras palabras, más que la toma de conciencia sobre lo que se decide, la posibilidad de decidir sobre aspectos sustanciales de la vida en comunidad, o la apropiación de espacios políticos, se decide sobre los recursos más básicos, los imprescindibles o los complementarios de la vida social y económica de la localidad; que, pensándolo bien, la mejora de su gestión sólo requiere de una voluntad política clara. Sin embargo, las posibles sugerencias de mayor calado, que requieren periodos de reflexión y cuestionamientos más profundos, son poco representativas o incluso inexistentes. $Y$ sin embargo, estas últimas son los que generan un reconocimiento mayor de la ciudadanía como parte del proceso, de la actuación participativa y de los procesos participados (análisis de influencia y cultura política de este estudio).

De esta forma, observamos en el estudio que las propuestas analizadas responden en su mayoría a una apuesta política clara por llevar adelante una gestión redistributiva más equitativa, que es posible con o sin la participación en los presupuestos. Por esta razón, nuestra pregunta se traslada ahora al trasfondo que nos han ofrecido los análisis. Ya que, nos preguntamos si tras el estudio de las propuestas se puede afirmar que ha existido una ampliación de ciudadanía entendida como la conciencia de "...la población a tener derechos: desde el derecho al acceso de bienes y servicios a como decidir (en diferentes grados) sobre la distribución del presupuesto", pues esto supone una diferencia significativa. Es decir, si esta toma de conciencia ha llevado a entender que las carencias no sólo están relacionadas con "... la privación de necesidades materiales, sino también como ausencia de capacidad para hacer uso de oportunidades y transformaciones en realizaciones, o como ausencia de poder para participar socialmente a nivel laboral, político y creativo" (Bonino, M., Bruera, S., 2005: 41-50). Los indicadores utilizados para el análisis de la capacidad de influencia y la cultura política nos hacen dudar sobre una realización parcial de estas finalidades.

\section{Bibliografía}

Allegretti. G y Herzberg. C. (2004). El 'retorno de las carabelas': los presupuestos participativos de Latinoamérica en el contexto europeo. Ansterdam, Transnational Institute. Fundación de Investigaciones Marxistas (TNI N²004/5).

Allegretti, G., (2011a). Aubague: desafíos de la democracia participativa. Le mode diplomatique (en español), año xv, nº 192.

Alledretti, G et all (2011b). Estudio comparativo de los presupuestos participativos en República Dominicana, España y Uruguay. CEDMA. Diputación de Málaga.

Barragán, V, Sanz, J.M y Romero, R (2014). Midiendo las experiencias participativas. El caso de los presupuestos participativos de Sevilla. En revista OIDLES, vol 8 número

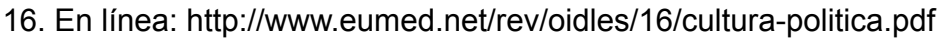

Bonino, M. y Bruera, S. (2005). Las mujeres en los presupuestos participativos. Reflexiones entorno a las experiencias de Montevideo, Porto Alegre y Recife. Montevideo: REPEM. 
Cabannes, Y (2004). "72 Respuestas a las 72 Preguntas Frecuentes sobre los Presupuestos Participativos". En Programa UN -HABITAT, Campana Global para una Mejor Gobernanza Urbana.

- (2008), Instrumentos de articulación entre Presupuesto Participativo y Ordenamiento Territorial, en IEPALA: Algo está pasando en Europa. 23-64, V jornadas Internacionales de Presupuestos Participativos, Sevilla.

Castoriadis C. (1995): "La democracia como procedimiento y como régimen", Leviatán: revista de hechos e ideas, $\mathrm{n}^{\circ} 64,65-84$.

- (2006) Castoriadis, C. Una sociedad a la deriva. Buenos Aires: Katz editores.

De Sousa Santos, B. (2003). Crítica a la razón indolente. Contra el desperdicio de la experiencia. Desclée. Bilbao.

Fedozzi, L. (1998). Esfera pública e ciudadania: a experiência do Orçamento Participativo de Porto Alegre. Esaios FEE, v19, n.2, 236-271, Porto Alegre.

Ganuza, E., y Álvarez, C., (2003), Ciudadanía y democracia: los presupuestos participativos, en Ganuza Fernandez, E. y Álvarez de Sotomayor, C. (coords). Democracia y presupuestos participativos. 13-37. Barcelona: Icarias.

Herrera, J (2005), La democracia en procesos participativos: principios, fundamentos y requisitos. En T. Villasante (co.). La Pedagogía de la Decisión. Construyendo ciudadanía/ 10, 87-114, Madrid: CIMAS.

- (2008) La reinvención de los Derecho Humanos. Sevilla: Atrapasueños Edit. y Univ. Pablo de Olavide.

Illich, I., (1973). La sociedad convivencial. Barcelona: Barral.

Max-Neef, M. A.(1994). Desarrollo a escala humana. Opciones de Futuro. Ed. Barcelona: Icarías.

Riechmann, J., (2006). ¿Cómo cambiar hacia sociedades sostenibles? Reflexiones sobre biomímesis y autolimitación. En Encina, J., y Barcena, I., Democracia ecológica. (Formas y experiencias de participación en la crisis ambiental). Democracia participativas/3, 73-92. Sevilla: Atrapasueños.

Rosa, M. Y Encina, J., (2004). "Democracias participativas desde la praxis local”. En Democracias participativas e intervención social comunitaria desde Andalucía (construyendo ciudadanía 5). Javier Encina (co.). Sevilla: ACNUR - Andalucía, Atrapasueños Edit. y Univ. Pablo de Olavide.

Rueda, S., (1997, 30 de junio). Habitabilidad y calidad urbana. En Ciudades para un futuro más sostenible: http://habitat.aq.upm.es/cs/

Sintomer, Y., (2001). ¿Empujar los límites de la democracia participativa? La democracia participativa en Europa y en Porto Alegre. Texto perteneciente a un seminario titulado: "O Orçamento Participativo visto pelos seus investigadores". Porto Alegre, 31/05-2/06/2001.

Souza, U. de, (2004). La experiencia de Porto Alegre y del estado de Rio Grande do Soul. Revista EI Viejo Topo, Número: 189-190, Barcelona.

Scharlock, R y Verdugo, M.A. (2003). Calidad de vida: manual para profesionales de la educación, la salud y servicios sociales. Madrid: Ministerio de trabajo y asuntos sociales. 